\title{
On Computing the Edge-balanced Index Sets of the Circle Union Graph F $(3, \mathrm{n})$
}

\author{
Yurong Ji \\ Henan Polytechnic University, Jiaozuo, China \\ E-mail: jiyurong@hpu.edu.cn \\ Jinmeng Liu \\ Henan College of Industry \& Information Technology, Jiaozuo, China \\ E-mail: xingfu0147@126.com
}

\begin{abstract}
On the basis of power-cycle nested network graph, the edge-balanced index sets of circle union graph $\mathrm{F}(3, \mathrm{n})$ graph were investigated. A new method of changing index is provided, simplifying the proving process. It reduced the difficulty of circle union graph $F(3, n)$ graph labeling because of the novel design of the basic graph and single-point sector subgraph. The results show that the edge-balanced index sets of circle union graph $F(3, n)$ graph. This paper has proved the existence of the edge-balanced index sets of one class of circle union graph, the computational formulas and the construction of the corresponding graphs are also provided.
\end{abstract}

Index Terms-Edge-friendly labeling, Edge-balance index set, the basic graph, circle union graph.

\section{INTRODUCTION}

Graph theory is an important branch of discrete mathematics which regards figure as the research object. B.M.Stewart introduced a theory in 1966 which use the vertices of graph and the label function of edges making the vertices of graph correspond to the edges, through the mapping function and then studies the characteristics and inherent characteristics of all kinds of figure. Its theory can be applied to the information engineering, communication network, computer science, economics and management, medicine and so on. Over the years many domestic and foreign researchers devote themselves to this area, and have access to a series of results.

In 1995, M.C.Kong and others defined the edge-balanced graph and strong edge-balanced graph in reference [1], and put forward two conjectures: Conjecture 1: All trees except $S_{n}, n$ is odd, are edge-balanced. Conjecture 2: All connected regular graphs except $K_{2}$ are edge-balanced. The reference [2] extends the concept of edge-balanced labeling to multigraphs and completely characterizes the edge-balanced multigraphs, and proves the two conjectures are true in the reference [1], at the same time proves the problem of decide a graph is edge-balanced does not belong to NP-hard. In 2008, Alexander and Harris studied the vertex -balanced index and friendly index sets of the figure in the literature [3-5].

Since 2009, author and her student Juan Lu solved the edge-balanced index sets of chain graph. In 2010, Ying Wang started the research about the edge-balanced index sets of the equal-cycle nested graph [7].From 2010, Hongjuan Tian[8] and others began the study of edge-balanced index sets of the nested graph with power-cycle.

In this paper, we completely determine the edge-balance index sets of circle union graph $F(3, n)$ by using the basic graph and recursive method. The computational formulas and the construction of the corresponding graphs are also provided.

The paper is organized as follows. In Section 2, we give the definitions that we shall consider. The lemmas are showed in Section 3. The main theorem is presented in Section 4. The final Section 5 contains the conclusion.

\section{PRELIMINARY RESULTS}

In graph theory, a graph $G$ is an ordered pair $(V(G), E(G))$ consists of vertex set $V(G)$ and edge set $E(G)$, together with an incidence function that associates with each edge of $G$ an ordered pair of vertices of $G$.

Definition 1 In graph $G$, let: $f: E(G) \rightarrow Z$ be an edge labeling function, that is to say, $\forall e \in E(G)$ to define $f(e)=0$ or 1 . The edge set labeled 0 or 1 is recorded as $E(0)$ and $E(1)$, using $e(0), e(1)$ to present the number of $E(0), E(1)$. According to the edge labeling $f$, we can define $f^{+}: V(G) \rightarrow\{0,1\}$. If $e_{x}(0)$ is more than $e_{x}(1)$, $f^{+}(x)=0$, the vertex $x$ is labeled 0 ; if $e_{x}(0)$ is less than $e_{x}(1), f^{+}(x)=1$, the vertex $x$ is labeled 1 ; otherwise, the vertex $x$ is unlabeled. $e_{x}(0)$ or $e_{x}(1)$ presents the radix number of the edge collection that the edges linked $x$ are labeled 0 or 1 . The radix number of the vertex set $V(0), V(1)$ is presented by $v(0)$ or $v(1)$. 
Definition 2 Let: $f: E(G) \rightarrow Z$ is an edge labeling function of $G$. If $|e(1)-e(0)| \leq 1, f$ is considered as an edge-friendly labeling of the graph $G$.

A graph $G$ is said to be edge-friendly if it admits an edge labeling $f$ such that $|e(1)-e(0)| \leq 1$. If, in addition, we also have $|v(1)-v(0)| \leq 1$, we call G edge-balanced. An edge-friendly graph could be far from being edge-balanced. To extend the study of edge-balancedness, we introduce the notion of an edge-balance index sets.

Definition 3: $\operatorname{EBI}(G)=\{|v(0)-v(1)|$ : the edge labeling $f$ is edge-friendly $\}$ is thought as the edge-balanced index set of the graph $G$, if there is an edge-friendly labeling $f$ in a graph $G$.

In other words, $\operatorname{EBI}(G)$ is the set of values that $|v(1)-v(0)|$ could attain as we go over all edge-friendly labelings of $G$.

Let $(H, x)$ denote a graph $H$ with a specific vertex.

Definition 4: Let $(\mathrm{H}, \mathrm{x})$ be a graph with a specified vertex $\mathrm{x}$, then the one-point union graph $\operatorname{Amal}((\mathrm{H}, \mathrm{x}), \mathrm{m})$ is defined by identifying the $\mathrm{x}$-vertex of $\mathrm{m}$ copies of $(\mathrm{H}, \mathrm{x})$.

Definition 5: Let $C_{n}=\left\{x, v_{1}, \ldots, v_{m-1}\right\}$ be a cycle. We call the one-point union graph Amal $\left(\left(C_{m}, x\right), n\right)$, denote by $F(m, n)$, a flower graph.

For simplicity we will denote it by $F(m, n)$. In this paper, we investigate $F(3, n)$. Let the vertices of the ith cycle of $F(3, n)$ be $x, v_{1}{ }^{i}, v_{2}{ }^{i}$, and assume that $x$ is the specific vertex used in the amalgamation.

Throughout this paper, $\bigcirc$ denote vertices labelled 0 ,

denote vertices labelled $1, \square$ denote vertices without label; — denote edges labelled 0 , denote edges labelled 1 .

\section{LEMMAS AND PROOF}

Lemma 1: For the flower graph $F(3,2)$, $\operatorname{EBI}(\mathrm{F}(3,2))=\{0\}$.

Proof: According to the definition of edge-friendly labeling, $|e(0)-e(1)| \leq 1$, we can get the edge-friendly labeling of the labeled graph by calculation.

By using enumeration, considering all possible edge assignment, we can get the edge-balance index of $F(3,2)$, $\operatorname{EBI}(F(3,2))=\{0\}$.

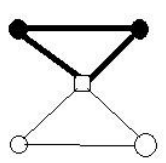

$|v(0)-v(1)|=0$

Fig.1

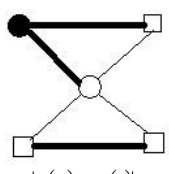

$|v(0)-v(1)|=0$ Fig.2

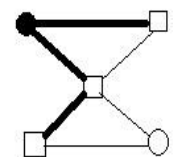

$|v(0)-v(1)|=0$

Fig.3
Lemma 2: For the flower graph $F(3,3)$, $\operatorname{EBI}(F(3,3))=\{0,1\}$

Proof: According to the definition of edge-friendly labeling, $|e(0)-e(1)| \leq 1$, we can get the edge-friendly labeling of the labeled graph by calculation.

By using enumeration, considering all possible edge assignment, we can get the edge-balance index of $\mathrm{F}(3,3)$, $\operatorname{EBI}(\mathrm{F}(3,3))=\{0,1\}$.

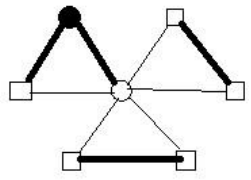

$|v(0)-v(1)|=1$

Fig.1

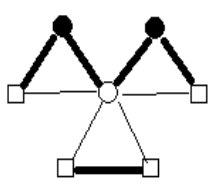

$|v(0)-v(1)|=0$

Fig. 2
Lemma 3: For the flower graph $F(3,4)$, $\operatorname{EBI}(\mathrm{F}(3,4))=\{0,1\}$.

Proof: According to the definition of edge-friendly labeling, $|e(0)-e(1)| \leq 1$, we can get the edge-friendly labeling of the labeled graph by calculation.

By using enumeration, considering all possible edge assignment, we can get the edge-balance index of $\mathrm{F}(3,4)$, $\operatorname{EBI}(\mathrm{F}(3,4))=\{0,1\}$.

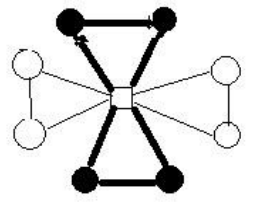

$|v(0)-v(1)|=0$

Fig.1

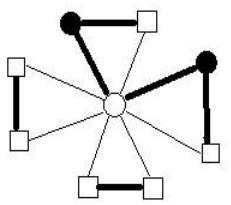

$|v(0)-v(1)|=1$
Lemma 4: For the flower graph $\mathrm{F}(3,5)$, $\operatorname{EBI}(\mathrm{F}(3,5))=\{0,1,2\}$.

Proof: According to the definition of edge-friendly labeling, $\mid e(0)-e(1) \leq 1$, we can get the edge-friendly labeling of the labeled graph by calculation.

By using enumeration, considering all possible edge assignment, we can get the edge-balance index of $\mathrm{F}(3,5)$, $\operatorname{EBI}(\mathrm{F}(3,5))=\{0,1,2\}$.

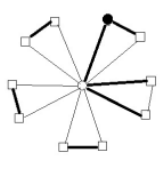

$|v(0)-v(1)|=0$

Fig. 1

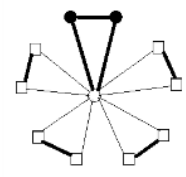

$|v(0)-v(1)|=1$

Fig. 2

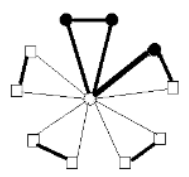

$|v(0)-v(1)|=2$

Fig.3
Lemma 5: For the flower graph $F(3,6)$, $\operatorname{EBI}(\mathrm{F}(3,6))=\{0,1,2\}$.

Proof: According to the definition of edge-friendly labeling, $|e(0)-e(1)| \leq 1$, we can get the edge-friendly labeling of the labeled graph by calculation. 
By using enumeration, considering all possible edge assignment, we can get the edge-balance index of $\mathrm{F}(3,6)$, $\operatorname{EBI}(F(3,6))=\{0,1,2\}$.

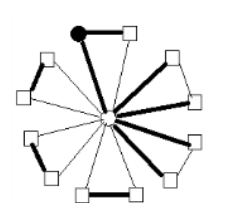

$|v(0)-v(1)|=0$

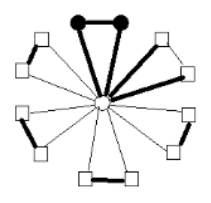

$|v(0)-v(1)|=1$

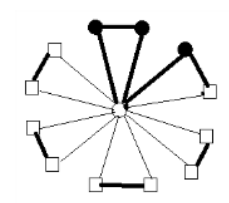

$|v(0)-v(1)|=2$

Fig.3
Fig.1

Fig.2

Lemma 6: For the flower graph $\mathrm{F}(3,7)$, $\operatorname{EBI}(\mathrm{F}(3,7))=\{0,1,2,3\}$.

Proof: According to the definition of edge-friendly labeling, $\mid e(0)-e(1) \leq 1$, we can get the edge-friendly labeling of the labeled graph by calculation.

By using enumeration, considering all possible edge assignment, we can get the edge-balance index of $\mathrm{F}(3,7)$, $\operatorname{EBI}(F(3,7))=\{0,1,2,3\}$.

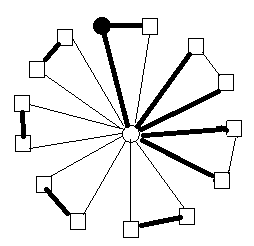

$|v(0)-v(1)|=0$

Fig.1

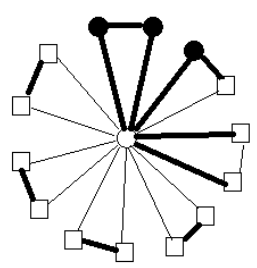

$|v(0)-v(1)|=2$

Fig.3

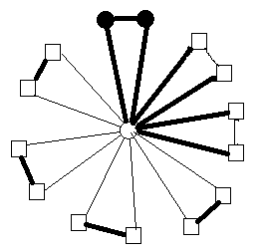

$|v(0)-v(1)|=1$

Fig. 2

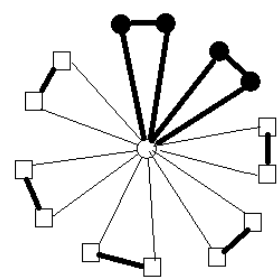

$|v(0)-v(1)|=3$

Fig.4
Lemma 7: For the flower graph $F(3,8)$, $\operatorname{EBI}(\mathrm{F}(3,8))=\{0,1,2,3\}$.

Proof: According to the definition of edge-friendly labeling, $|e(0)-e(1)| \leq 1$, we can get the edge-friendly labeling of the labeled graph by calculation.

By using enumeration, considering all possible edge assignment, we can get the edge-balance index of $\mathrm{F}(3,8)$, $\operatorname{EBI}(F(3,8))=\{0,1,2,3\}$.

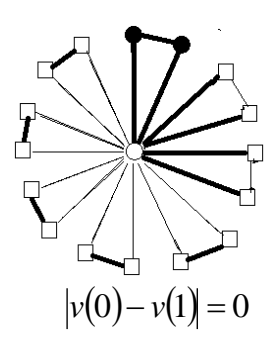

Fig.1

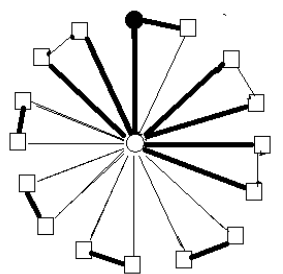

$|v(0)-v(1)|=1$
Fig.2

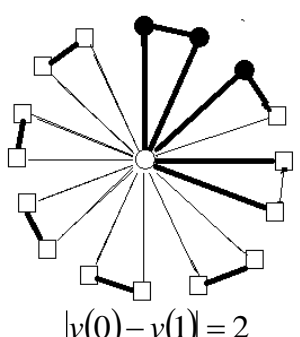

Fig. 3

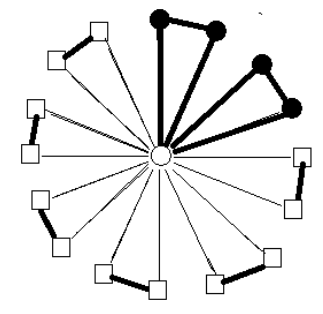

$|v(0)-v(1)|=3$

Fig.4
Lemma 8: For the flower graph $F(3,9)$, $\operatorname{EBI}(F(3,9))=\{0,1,2,3,4\}$.

Proof: According to the definition of edge-friendly labeling, $|e(0)-e(1)| \leq 1$, we can get the edge-friendly labeling of the labeled graph by calculation.

By using enumeration, considering all possible edge assignment, we can get the edge-balance index of $\mathrm{F}(3,9)$, $\operatorname{EBI}(\mathrm{F}(3,9))=\{0,1,2,4\}$.

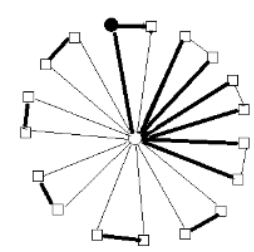

$|v(0)-v(1)|=0$

Fig.1

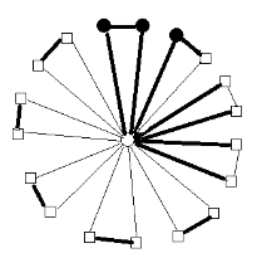

$|v(0)-v(1)|=2$

Fig.3

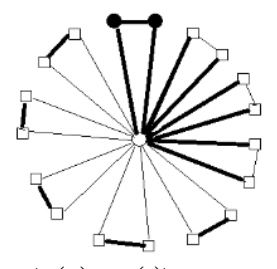

$|v(0)-v(1)|=1$

Fig. 2

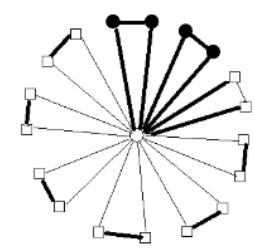

$|v(0)-v(1)|=3$

Fig.4

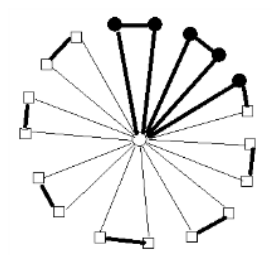

$|v(0)-v(1)|=4$

Fig. 5

Then, we can get the following lemma.

Lemma 9: When $2 \leq n \leq 9$,

$$
\operatorname{EBI}(F(3, n))= \begin{cases}\left\{0,1, \cdots, \frac{n}{2}-1\right\}, & n=2,4,6,8 \\ \left\{0,1, \cdots, \frac{n-1}{2}\right\}, & n=3,5,7,9\end{cases}
$$




\section{THEOREM}

Theorem 1: For $n \geq 2$,

$$
\operatorname{EBI}\left(F(3, n)= \begin{cases}\left\{0,1,2, \ldots, \frac{n}{2}-1\right\} & \text { if } n \text { is even } \\ \left\{0,1,2, \ldots, \frac{n-1}{2}\right\} & \text { if } n \text { is odd }\end{cases}\right.
$$

\section{Proof:}

Case 1: Given $n \geq 2$ and $n$ is even.

Let $v_{1}^{j} v_{2}^{j}(1 \leq j \leq n)$ and $x v_{1}^{k}\left(1 \leq k \leq \frac{n}{2}\right)$ be 1-edges, and the remaining edges be 0 -edges. We call the labeled graph be the basic graph 1 . It follows immediately that $f$ is friendly and $v(0)=1, v(1)=\frac{n}{2}$. Then $\frac{n}{2}-1 \in \operatorname{EBI}(F(3, n))$.

Firstly, we prove $\max \{\operatorname{EBI}(F(3, n))\}=\frac{n}{2}-1$.

In the basic graph 1, we investigate the edge- balance index change when exchanging arbitrary 1-edge and 0 -edge. It can be divided five cases to discuss.

(1) Any one of the 1-edge $x v_{1}^{i}\left(1 \leq i \leq \frac{n}{2}\right)$ and 0-edge exchange, $v(0)$ and $v(1)$ have no change. Thus $|v(1)-v(0)|$ remains unchanged.

(2) Any one of the 1-edge $v_{1}^{i} v_{2}^{i}\left(1 \leq i \leq \frac{n}{2}\right)$ and 0-edge $x v_{2}^{i}$ in the same cycle exchange, $v(0)$ constant, $\quad v(1)$ is reduced. Thus $|v(1)-v(0)|$ is reduced.

(3) Any one of the 1-edge $v_{1}^{i} v_{2}^{i}\left(1 \leq i \leq \frac{n}{2}\right)$ and 0-edge except $x v_{2}^{i}$ exchange, $v(1)$ constant, $v(0)$ is on the increase. Thus $|v(1)-v(0)|$ is reduced.

(4) Any one of the 1-edge $v_{1}^{i} v_{2}^{i}\left(\frac{n}{2}+1 \leq i \leq n\right)$ and 0-edge $x v_{1}^{i}$ or $x v_{2}^{i}$ in the same cycle exchange, $v(1)$ constant, $v(0)$ is on the increase. Thus $|v(1)-v(0)|$ is reduced.

(5) Any one of the 1-edge $v_{1}^{i} v_{2}^{i}\left(\frac{n}{2}+1 \leq i \leq n\right)$ and the same cycle 0 -edge except $x v_{1}^{i}$ and $x v_{2}^{i}$ exchange, $v(1)$ and $v(0)$ are increasing by 1 and 2 . Thus $|v(1)-v(0)|$ is reduced.

In summary, It is obvious that the exchanging must bring $|v(1)-v(0)|$ decreases or remain unchanged. Thus $\max \{\operatorname{EBI}(F(3, n))\}=\frac{n}{2}-1$.

Next we prove $\left\{0,1,2, \ldots, \frac{n}{2}-1\right\} \subseteq \operatorname{EBI}(F(3, n))$

Let $A \leftrightarrow B$ denote 1 -edge $A$ and 0-edge $B$ exchange their label. Each step is on the basis of the above step. The exchange in turn is as follows.

$$
\begin{array}{cr}
v_{1}^{1} v_{2}^{1} \leftrightarrow x v_{2}^{1} & |v(1)-v(0)|=\frac{n}{2}-2 \\
x v_{2}^{1} \leftrightarrow v_{1}^{2} v_{2}^{2} & |v(1)-v(0)|=\frac{n}{2}-3 \\
\ldots & \ldots \\
x v_{2}^{k} \leftrightarrow v_{1}^{k} v_{2}^{k} & |v(1)-v(0)|=\frac{n}{2}-k-1 \\
\ldots & \cdots \\
x v_{2}^{\frac{n}{2}-1} \leftrightarrow v_{1}^{\frac{n}{2}-1} v_{2}^{\frac{n}{2}-1} & |v(1)-v(0)|=0
\end{array}
$$

Thus $\operatorname{EBI}(F(3, n))=\left\{0,1,2, \ldots, \frac{n}{2}-1\right\} \quad(n$ is even $)$.

Case2: Given $n \geq 2$ and $n$ is odd.

Let $v_{1}^{j} v_{2}^{j}(1 \leq j \leq n)$ and $x v_{1}^{k}\left(1 \leq k \leq \frac{n+1}{2}\right)$ be 1-edges, and the remaining edges be 0 -edges. We call the labeled graph be the basic graph 2 . It follows immediately that $f \quad$ is friendly and $\quad v(0)=1, v(1)=\frac{n+1}{2}$. Then $\frac{n-1}{2} \in \operatorname{EBI}(F(3, n))$.

Firstly, we prove $\max \{\operatorname{EBI}(F(3, n))\}=\frac{n-1}{2}$.

In the basic graph 2, we investigate the edge- balance index change when exchanging arbitrary 1-edge and 0 -edge. It can be divided five cases to discuss.

(1) Any one of the 1-edge $x v_{1}^{i}\left(1 \leq i \leq \frac{n+1}{2}\right)$ and 0-edge exchange, $v(0)$ and $v(1)$ have no change. Thus $|v(1)-v(0)|$ remains unchanged.

(2) Any one of the 1-edge $v_{1}^{i} v_{2}^{i}\left(1 \leq i \leq \frac{n+1}{2}\right)$ and 0-edge $x v_{2}^{i}$ in the same cycle exchange, $v(0)$ constant, $\quad v(1)$ is reduced. Thus $|v(1)-v(0)|$ is reduced.

(3) Any one of the 1-edge $v_{1}^{i} v_{2}^{i}\left(1 \leq i \leq \frac{n+1}{2}\right)$ and 0-edge except $x v_{2}^{i}$ exchange, $v(1)$ constant, $v(0)$ is on the increase. Thus $|v(1)-v(0)|$ is reduced.

(4) Any one of the 1-edge $v_{1}^{i} v_{2}^{i}\left(\frac{n+3}{2} \leq i \leq n\right)$ and 0-edge $x v_{1}^{i}$ or $x v_{2}^{i}$ in the same cycle exchange, $v(1)$ constant, $v(0)$ is on the increase. Thus 
$|v(1)-v(0)|$ is reduced.

(5) Any one of the 1-edge $v_{1}^{i} v_{2}^{i}\left(\frac{n+3}{2} \leq i \leq n\right)$ and the same cycle 0 -edge except $x v_{1}^{i}$ and $x v_{2}^{i}$ exchange, $v(1)$ and $v(0)$ are increasing by 1 and 2 . Thus $|v(1)-v(0)|$ is reduced.

In summary, It is obvious that the exchanging must bring $|v(1)-v(0)|$ decreases or remain unchanged. Thus $\max \{\operatorname{EBI}(F(3, n))\}=\frac{n-1}{2}$.

$$
\text { Next we prove }\left\{0,1,2, \ldots, \frac{n-1}{2}\right\} \subseteq E B I(F(3, n))
$$

Let $A \leftrightarrow B$ denote 1-edge $A$ and 0 -edge $B$ exchange their label. Each step is on the basis of the above step. The exchange in turn is as follows.

$$
\begin{array}{cr}
v_{1}^{1} v_{2}^{1} \leftrightarrow x v_{2}^{1} & |v(1)-v(0)|=\frac{n}{2}-3 \\
x v_{2}^{1} \leftrightarrow v_{1}^{2} v_{2}^{2} & |v(1)-v(0)|=\frac{n-5}{2} \\
\ldots & \ldots \\
x v_{2}^{k} \leftrightarrow v_{1}^{k} v_{2}^{k} & |v(1)-v(0)|=\frac{n-1-2 k}{2} \\
\ldots & \ldots \\
x v_{2}^{\frac{n-3}{2}} \leftrightarrow v_{1}^{\frac{n-3}{2}} v_{2}^{\frac{n-3}{2}} & |v(1)-v(0)|=1
\end{array}
$$

Now we only need to prove $0 \in \operatorname{EBI}(F(3, n))$. Assume a new label $f$, Let $x v_{1}^{i}\left(1 \leq i \leq \frac{n-1}{2}\right)$, $v_{1}^{k} v_{2}^{k}\left(\frac{n-1}{2} \leq k \leq n\right)$ and $x v_{2}^{j}\left(1 \leq j \leq \frac{n-3}{2}\right)$ be 1-edges, and the remaining edges be 0 -edges. Thus $|v(1)-v(0)|=0$. Thus $\operatorname{EBI}(F(3, n))=\left\{0,1,2, \ldots, \frac{n-1}{2}\right\} \quad(n$ is odd $)$.

The proof is completed.

\section{CONCLUSION}

Based on power-cycle nested network graph, using the new designs about the graph, analysis of induction, the edge-balanced index sets of Circle union $F(3, n)$ graph were investigated. we showed the proof of the computational formula, at the same time giving the construction of the corresponding graphs. The resulting work using the same method will be investigated in a future paper.

\section{REFERENCES}

[1] Kong M, Sin-Min Lee. On Edge-Balanced Graphs [J], Graph Theory, Combinatoric and Algorithms, V.1, 711 -722(1995).
[2] B.L.Chen, K.C. Huang and Shi-Shen Liu. On edge-balanced multigraphs, Journal of Combinatorial Mathematics and Combinatorial Computing, 42 (2002),177-185.R. Nicole.

[3] AleLee and Ho Kuen Ng. On The Balance Index Set of Graphs [J]. Journal of Combina-torial Mathematics and Combinatorial Computing. 2008(66): 135-150.

[4] Harris Kwong, Sin-Min Lee and Ho Kuen $\mathrm{Ng}$. On Friendly Index Sets of 2-Regular Graphs, Discrete Mathematics, 2008, 308: p. 5522-5532.

[5] Ebrahim Salehi and Sin-Min Lee, On Friendly Index Sets of Trees, Congressus Numeran-tium, 2006, 178: p. 173-183.

[6] Juan Lu and Yuge Zheng: On the edge-balance index sets of $B(n)$, Proceedings of the Jangjeon Mathematical Society, 12(1), 2009, 37-44.

[7] Ying Wang, Yuge Zheng and Sin-Min Lee: On the quick construction of all edge-balance index sets of $C_{n} \times P_{2}$ $(n \equiv 2,3(\bmod 4)) \quad[\mathrm{J}]$. Proceedings of the Jangjeon Mathematical Society. 2010(13), No.3: 387-393.

[8] Yuge Zheng, Hongjuan Tian, On the Edge-Balance Index Sets of the Power Circle Nested Graph C2m $\times$ Pm2 (m $\equiv$ 0(mod 2)), Advanced Science Letters, Volume 7 2012, pp. 534-536(3)

[9] WANG Y, ZHENG Y G, ADIGA C, et al. On the edge-balance index sets of $\mathrm{N}$ cycles three nested graph $(\mathrm{N}=0, \quad 1, \quad 2(\bmod 6)) \quad[\mathrm{J}]$. Advanced Studied in Contemporary Mathematics, 2011, 21(1): 85-93.

[10] YAO J, ZHENG Y G. On the quick construction of all edge-balance index sets of the graph $\mathrm{C} n \times \mathrm{P} 5[\mathrm{C}] / /$ Consumer Electronics, Communications and Networks (CEC Net). 2011 International Conference on. IEEE. Xianning, China: IEEE Press, 2011: 4227-4230.

[11] Zheng Y, Tian H. On the Edge-Balance Index Sets of the Power Circle Nested Graph C2m Pm2 $(\operatorname{m~} 0(\bmod 2))[\mathrm{J}]$. Advanced Science Letters, 2012, 7(1): 534-536.

[12] Yuge Zheng, Jingjing Yao. The Edge-balance Index Sets of Nested Graph with the Unlimited Paths and Equa Circles (I) [J]. Journal of Shnahai Jiaotong University (Science)., 2013, 47(07): 1160-1163.

[13] Zheng Y, Tian H. On the edge-balance index sets of a classes of nested network graph[C]//Uncertainty Reasoning and Knowledge Engineering (URKE), 2012 2nd International Conference on. IEEE. Jakarta, Indonesia: IEEE Press, 2012: 170-174.

[14] Jinmeng Liu, Tou Hou, Yuge Zheng. The Edge-balance Inedx Sets of two Classes of Nested Network Graph [J].Computer Science, 2015, 42(3):245-251.

[15] Liu J, Zheng Y. Edge-balanced Index Sets of the Nested Graph with Power-cycleC5mxPm5 (I) [J]. International Journal of Modern Education and Computer Science (IJMECS), 2013, 5(9): 53.

\section{Authors' Profiles}

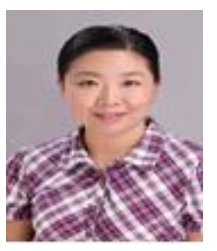

Yurong Ji: Associate Professor of Henan Polytechnic University, interested in Graph theory and Combinatorial mathematics 


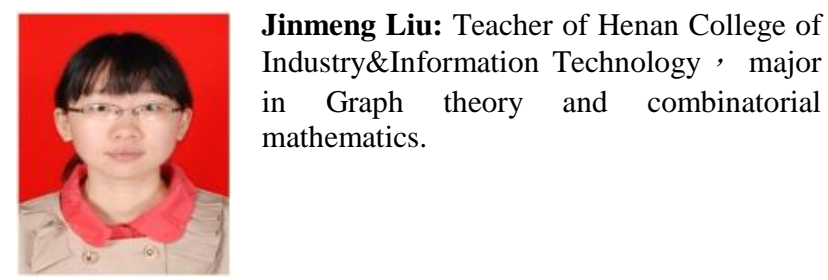

How to cite this paper: Yurong Ji, Jinmeng Liu,"On Computing the Edge-balanced Index Sets of the Circle Union Graph F (3, n)", International Journal of Modern Education and Computer Science(IJMECS), Vol.8, No.3, pp.22-27, 2016.DOI: $10.5815 /$ ijmecs.2016.03.03 